\title{
Positive correlation between blood reflux in Schlemm's canal and the decrease of intraocular pressure after selective laser trabeculoplasty in primary open-angle glaucoma
}

\author{
JIE-LING GONG ${ }^{*}$, SAMER ABDO AL-WESABI ${ }^{*}$, YIN ZHAO and HONG ZHANG \\ Department of Ophthalmology, Tongji Hospital, Tongji Medical College, \\ Huazhong University of Science and Technology, Wuhan, Hubei 430030, P.R. China
}

Received October 26, 2017; Accepted March 2, 2018

DOI: $10.3892 /$ etm.2018.6051

\begin{abstract}
The aim of the present study was to investigate the association between blood reflux in Schlemm's canal (SC) and the decrease of intraocular pressure (IOP) after selective laser trabeculoplasty (SLT) in primary open-angle glaucoma (POAG). To verify this, 35 eyes from 25 POAG patients were enrolled. All eyes underwent $360^{\circ}$ whole-circle gonioscopy via a three-mirror lens, prior to undergoing $360^{\circ} \mathrm{SLT}$. The four quadrants of the examined eye were individually compressed by the lens, and the presence of blood reflux in SC after removal of the pressure was recorded. Eyes with no blood reflux in any quadrant were assigned to the negative group, while the others were assigned to the positive group. Patients were evaluated at baseline, at 1 and 2 weeks, and at 1, 3 and 6 months after SLT. The results indicated that in the reflux-positive (21 eyes) and -negative (14 eyes) group, a significant IOP decrease was seen at 1 and 2 weeks, and at 1 and 3 months after SLT as compared with that pre-SLT IOP $(\mathrm{P}<0.01)$. In the negative group, the IOP at 6 months after SLT was not significantly different $(\mathrm{P}>0.05)$, while the positive group still exhibited a significant decrease in IOP compared with that at baseline $(\mathrm{P}<0.01)$. The positive group also presented with a greater decline in IOP at 1, 3 and 6 months compared with that in the negative group $(\mathrm{P}<0.05)$. A positive correlation between the number of quadrants with blood reflux in SC and the percentage decrease in IOP after SLT was identified in the positive group $(\mathrm{P}<0.05)$. In conclusion, blood reflux in $\mathrm{SC}$ was
\end{abstract}

Correspondence to: Dr Yin Zhao or Dr Hong Zhang, Department of Ophthalmology, Tongji Hospital, Tongji Medical College, Huazhong University of Science and Technology, 1095 Jiefang Ave, Wuhan, Hubei 430030, P.R. China

E-mail: zhaoyin85@hust.edu.cn

E-mail: dr_zhanghong@vip.163.com

${ }^{*}$ Contributed equally

Key words: blood reflux, Schlemm's canal, selective laser trabeculoplasty, intraocular pressure, primary open-angle glaucoma positively correlated with the decrease in IOP after SLT in POAG (Trialapproval number, K-2014-013).

\section{Introduction}

Primary open angle glaucoma (POAG) is an optic neuropathy in which high intraocular pressure (IOP) may result in optic nerve damage, subsequently leading to peripheral or central visual field loss (1). The increased IOP in POAG is caused by an increase in aqueous outflow resistance in the drainage pathways, rather than by excess secretion of aqueous humor (2). In the classical aqueous outflow pathway of normal eyes, $75 \%$ of the outflow resistance is located in the trabecular meshwork (TM) and $25 \%$ is located in Schlemm's canal (SC) (3).

Surgical treatment of POAG is currently mostly aimed at decreasing the abnormal outflow resistance, with different surgical approaches focused on different sites of the drainage pathway. Selective laser trabeculoplasty (SLT) was first presented by Latina and Park (4) in 1995. It was designed to selectively target pigmented cells in the TM in order to lower the resistance in the TM, while sparing adjacent cells and tissues from thermal damage and maintaining the TM architecture $(5,6)$. It is an effective, non-invasive IOP-lowering procedure for patients with POAG in whom the natural aqueous outflow system is still intact. Certain studies have revealed that SLT results in a $>20 \%$ reduction in IOP $(7,8)$. However, SLT treatment is not uniformly effective in all eyes $(9,10)$. Identifying patients who are most likely to benefit from the application of SLT is crucial.

$\mathrm{SC}$ is comprised of endothelial cells surrounded by connective tissue, similar to a vein. In 1914, Salzmann first described that blood reflux in SC was seen ongonioscopy after applying gentle pressure on the globe of normal eyes (11). This blood reflux may reflect the drainage resistance. Previous studies have indicated that, if blood reflux into the canal is clearly seen through the TM of POAG patients, better results may be achieved by canaloplasty $(12,13)$. However, to date, no method for the evaluation of the functional status of SC prior to SLT has been described.

The aim of the present study was to investigate the association between the blood reflux in SC observed during gonioscopy and the decrease of IOP after SLT, in order to 
provide clinical insight into its application for the prediction of SLT outcomes.

\section{Materials and methods}

Subjects. The present study adhered to the tenets of the Declaration of Helsinki. The study was approved by the institutional ethics committees of Tongji Hospital, Huazhong University of Science and Technology (Wuhan, China; Trial approval no. K-2014-013). Written informed consent was obtained from each patient prior to the start of the study.

This prospective cohort study sequentially recruited patients from the ophthalmology clinics of Tongji Hospital (Huazhong University of Science and Technology, Wuhan, China), from March 2015 to November 2015. Patients with severe refractive medium opacity, high intraocular pressure, dense trabecular pigmentation preventing clear observation of blood reflux, severe systemic and mental disease, or who had undergone any ocular surgery were excluded.

Surgical technique. All eyes underwent gonioscopy prior to SLT. During gonioscopy, slight pressure was applied to the viewed quadrant to raise the episcleral venous pressure and draw the blood back into SC. Depending on the presence (positive group) or absence (negative group) of blood reflux, patients were divided into two groups (Fig. 1). The positive group was comprised of 21 eyes and the negative group included 14 eyes. In the positive group, the observation of blood reflux was recorded in quadrants 1, 2, 3 and 4, based on the appearance of blood on the trabeculum in each quadrant seen using the Haag-Streit-Goldmann three-mirror lens.

To estimate the group size, a pilot study was performed for measuring the IOP at 1 month after SLT in 10 eyes of 10 patients with uncontrolled POAG. The standard deviation of the reduction of the IOP in this group was $3.90 \mathrm{mmHg}$. The present study aimed to measure a difference in IOP of $4 \mathrm{mmHg}$ among the groups. To achieve a two-tailed $\alpha=0.05$ and a statistical power of $80 \%$, at least 12 patients per group were required. Considering a compliance rate of $90 \%$, the present study eventually enrolled 35 eyes of 25 patients with POAG, including 21 eyes in the positive group and 14 eyes in the negative group. The sample size in the two groups was suitable for the statistical equation and was acceptable according to the requirements of the statistics. The essential information and the baseline (pre-SLT) IOP of the patients are presented in Table I.

All patients underwent a single $360^{\circ}$ SLT session that was performed by a single surgeon (HZ). After discussion, the investigators agreed on a standardized laser technique to minimize the variation in power, number and location of laser spots. An initial laser energy of $0.6 \mathrm{~mJ}$ was applied and was then adjusted until bubble formation was just visible. After SLT, the subjects continued with the same IOP-lowering treatment, and were prescribed Pranoprofen eye drops (Pranopulin, Senju Pharmaceutical Co., Ltd., Osaka, Japan) three times a day for 5 days (14).

After the surgery, the IOP was measured at 1 and 2 weeks, and at 1,3 and 6 months after SLT. All IOP measurements were performed via non-contact tonometry by a single investigator (JG) who was blinded to the specific group of the patients. The measurements were taken at least three times in every eye
A

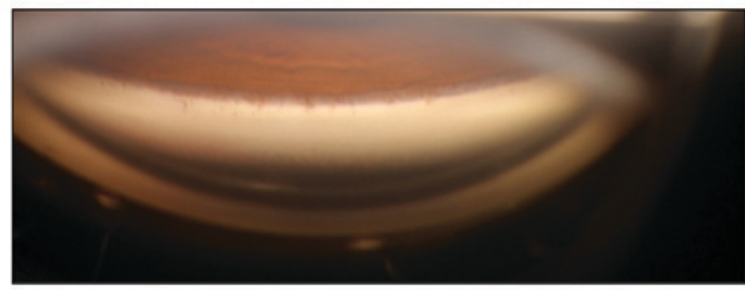

B

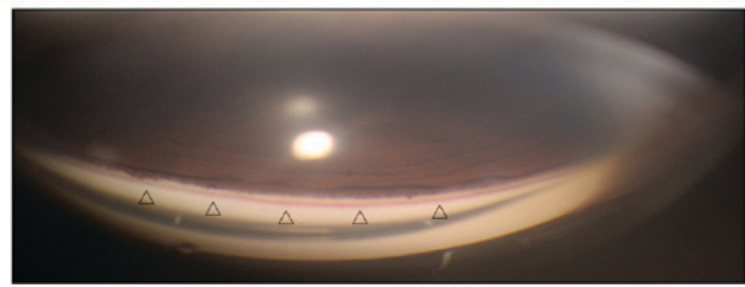

Figure 1. Gonioscopic view of the chamber angle with iris processes. (A) In the negative group, blood reflux in SC was absent after removal of the pressure from the lens at the viewed quadrant. (B) In the positive group, blood is observed in the SC removal of the pressure from the lens at the viewed quadrant. The two images were taken at the same quadrant from different eyes. $\Delta$ indicates blood in the SC. SC, Schlemm's canal.

and the average was taken. Patients returned for follow-up at approximately the same time of day $(9-11 \mathrm{am})$ to minimize the effect of diurnal IOP fluctuation. The number of medications was tapered according to the level of IOP measured after SLT in order to maintain the target IOP.

Statistical analysis. Statistical analysis was all performed using SPSS version 19 (IBM Corp., Armonk, NY, USA) and values for the study population were expressed as the mean \pm standard deviation. Intra-group and inter-group comparisons of the IOP were performed using generalized estimation equations (GEE), which take the correlation between right and left eye into consideration (15). Spearman's correlation was used to evaluate the association between blood reflux quadrants and IOP after SLT.

\section{Results}

SLT causes an obvious decrease in IOP. The pre-operative mean IOP served as the baseline IOP, and was $20.2 \pm 4.2 \mathrm{mmHg}$ in the positive group and $21.0 \pm 5.3 \mathrm{mmHg}$ in the negative group (Table I). Compared with the baseline, the positive and the negative group exhibited a decrease in IOP at 1 and 2 weeks, and at 1 and 3 months after the SLT. In the negative group, the IOP at 6 months after SLT was not significantly different from that at baseline $(\mathrm{P}=0.066)$, while the positive group still exhibited a significant decrease in IOP compared with the baseline $(\mathrm{P}<0.001$; Table II).

Blood reflux in SC is associated with a greater decrease in IOP after SLT. The decrease in IOP was different between the two groups. The two groups were also different in terms of age and gender. When the age and gender were not adjusted, the positive group only exhibited a significantly greater decrease in IOP compared with that in the negative group at 1 and 3 months after SLT $(\mathrm{P}=0.024$ and 0.018 , respectively). 
Table I. Baseline data in all patients.

\begin{tabular}{lccc}
\hline Parameter & Positive group $(\mathrm{n}=21)$ & Negative group $(\mathrm{n}=14)$ & P-value \\
\hline Age (years) & $44.60 \pm 15.0$ & $51.0 \pm 11.9$ & 0.428 \\
Right eye n (\%) & $7(34)$ & $5(36)$ & 0.889 \\
Left eye n (\%) & $14(66)$ & $9(64)$ & $/$ \\
Pre-SLT IOP (mmHg) & $20.2 \pm 4.2$ & $21.0 \pm 5.3$ & 0.792 \\
Blood reflux quadrants (N) & $2.2 \pm 0.8$ & $/$
\end{tabular}

Values are expressed as the mean \pm standard deviation or the percentage. IOP, intraocular pressure; SLT, selective laser trabeculoplasty; $\mathrm{n}$, number of eyes; $\mathrm{N}$, number of quadrants; /, inconclusive results.

Table II. Data of IOP in the positive group and the negative group at different time-points.

\begin{tabular}{|c|c|c|c|c|c|c|}
\hline \multirow[b]{2}{*}{ Time-point } & \multicolumn{3}{|c|}{ Positive group } & \multicolumn{3}{|c|}{ Negative group } \\
\hline & IOP (mmHg) & $\beta(95 \% \mathrm{CI})$ & P-value ${ }^{a}$ & IOP (mmHg) & $\beta(95 \% \mathrm{CI})$ & P-value ${ }^{a}$ \\
\hline Pre & $20.2 \pm 4.2$ & l & / & $21.0 \pm 5.3$ & / & 1 \\
\hline $1 \mathrm{w}$ & $15.1 \pm 4.2$ & $-5.095(-6.678,-3.513)$ & $<0.001$ & $16.1 \pm 3.5$ & $-4.929(-7.140,-2.717)$ & $<0.001$ \\
\hline $2 \mathrm{w}$ & $14.8 \pm 3.3$ & $-5.462(-7.411,-3.513)$ & $<0.001$ & $16.2 \pm 2.6$ & $-4.800(-7.100,-2.500)$ & $<0.001$ \\
\hline $1 \mathrm{~m}$ & $13.9 \pm 2.7$ & $-6.310(-8.524,-4.095)$ & $<0.001$ & $16.8 \pm 3.6$ & $-4.236(-6.909,-1.563)$ & 0.002 \\
\hline $3 \mathrm{~m}$ & $13.7 \pm 2.1$ & $-6.538(-8.458,-4.618)$ & $<0.001$ & $15.8 \pm 2.4$ & $-5.236(-7.910,-2.562)$ & $<0.001$ \\
\hline $6 \mathrm{~m}$ & $15.1 \pm 3.4$ & $-5.131(-6.658,-3.603)$ & $<0.001$ & $17.8 \pm 3.0$ & $-3.309(-6.843,-0.225)$ & 0.066 \\
\hline
\end{tabular}

${ }^{a}$ Compared with the pre IOP. Pre, pre-SLT (the pre-SLT IOP severed as the baseline in each group), CI, confidence interval; w, week; $\mathrm{m}, \mathrm{month}(\mathrm{s})$; SLT, selective laser trabeculoplasty; $\beta$, regression coefficient.

Table III. Comparison between the IOP of the positive and the negative group.

\begin{tabular}{|c|c|c|c|c|}
\hline \multirow[b]{2}{*}{ Time-point } & \multicolumn{2}{|c|}{ Unadjusted } & \multicolumn{2}{|c|}{ Adjusted } \\
\hline & $\beta(95 \% \mathrm{CI})$ & P-value & $\beta(95 \% \mathrm{CI})$ & P-value \\
\hline Pre & $-0.762(-4.862,3.338)$ & 0.716 & $-0.890(-4.837,3.056)$ & 0.659 \\
\hline $1 \mathrm{w}$ & $-0.929(-3.753,1.895)$ & 0.519 & $-1.148(-3.665,1.369)$ & 0.371 \\
\hline $2 \mathrm{w}$ & $-1.424(-3.585,0.737)$ & 0.197 & $-1.589(-3.696,0.518)$ & 0.139 \\
\hline $1 \mathrm{~m}$ & $-2.836(-5.290,-0.382)$ & 0.024 & $-2.909(-5.238,-0.580)$ & 0.014 \\
\hline $3 \mathrm{~m}$ & $-2.064(-3.777,-0.351)$ & 0.018 & $-2.149(-3.836,-0.462)$ & 0.013 \\
\hline $6 \mathrm{~m}$ & $-2.625(-5.484,0.234)$ & 0.072 & $-2.863(-5.667,-0.059)$ & 0.045 \\
\hline
\end{tabular}

$\beta / P$ : The influence factor age and gender have been adjusted using GEE. $\beta$, regression coefficient; Pre, pre-SLT (the pre-SLT IOP severed as the baseline in each group); CI, confidence interval; w, week; m, month(s); SLT, selective laser trabeculoplasty.

After those two variables were adjusted by GEE, the positive group exhibited a greater decrease in IOP after SLT at 1, 3 and 6 months $(\mathrm{P}=0.014,0.013$ and 0.045 , respectively; Table III).

Positive correlation between the number of quadrants with blood reflux and IOP decrease after SLT. After $360^{\circ}$ SLT, the reflux-positive group clearly presented with a more marked reduction in IOP than the negative group. To further investigate this, a correlation analysis was used to analyze the association between the extent of decrease in IOP and the number of quadrants in which blood reflux appeared in SC. (Fig. 2). At 3 and 6 months, a strongly positive correlation between the number of quadrants with blood reflux in SC and the percentage decrease in IOP was identified $(\mathrm{P}=0.037$ and 0.020 , respectively).

\section{Discussion}

In the present prospective observational study, POAG patients with blood reflux in SC exhibited a greater decrease in IOP 

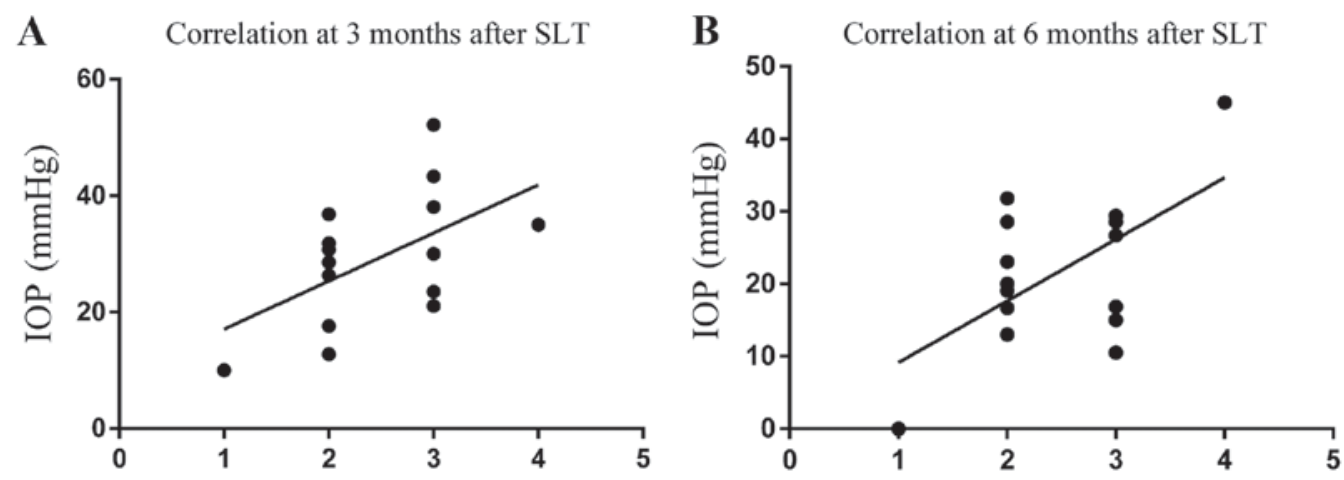

Figure 2. In the positive group, the number of quadrants with blood reflux was positively correlated with the decrease in IOP after SLT. This correlation was identified at (A) 3 months ( $\mathrm{P}=0.037)$ and (B) 6 months $(\mathrm{P}=0.020)$ after the SLT. The abscissa refers to the numbers of the quadrants with blood reflux in Schlemm's canal. SLT, selective laser trabeculoplasty; IOP, intraocular pressure.

after SLT and the number of quadrants with blood reflux was positively correlated with the decrease.

IOP is regarded as an important factor in the progression of POAG (16). Almost $70 \%$ of the aqueous hum or outflow occurs via the anterior chamber to the TM, SC and collector channels, entering the systemic venous circulation through the episcleral veins (17). Studies on the aqueous outflow pathways have revealed that, in humans, $75 \%$ of the resistance to the aqueous humor outflow is localized to the $\mathrm{TM}$, and $25 \%$ to $\mathrm{SC}$, the outer wall of SC or the tissue surrounding SC $(18,19)$. As an abnormal increase of the outflow resistance may cause an increase in IOP, the development of a novel surgical treatment for glaucoma must involve the search for how to reduce IOP, and more specifically, to reduce the outflow resistance in glaucoma.

Numerous surgeries are available that target the sites of the outflow resistance, including trabeculectomy, viscocanalostomy, argon laser trabeculoplasty and SLT (5). Surgeries designed to incise or remove the abnormal TM in glaucoma, e.g., trabeculectomy, address the abnormal resistance in the TM, while other surgeries target SC by unroofing or expanding the canal, e.g., viscocanalostomy $(20,21)$. SLT is based on the principle of selective thermolysis, in which laser energy applied to the TM selectively targets pigmented cells without causing significant collateral thermal damage (6). In the present study, SLT resulted in a 20.1-30.4\% reduction in IOP. Thus, the effect exhibited a great variation in patients with POAG. Numerous factors have been investigated, but none of them has been identified as a significant predictor of successful treatment, including age, sex, race, glaucoma type, TM pigmentation, angle grade, lens status and central corneal thickness (22). As the SLT mainly reduces the resistance located in the TM, it is suspected that the resistance originating from SC may be an important factor for determining the difference between the positive and the negative group.

Blood is ordinarily visible in the SC by gonioscopy. The three most common reasons are ocular hypotony, high episcleral venous pressure and compression of the episcleral veins by the examiner's goniolens $(23,24)$. Grieshaber $(25)$ observed blood reflux into SC in eyes with POAG by using provocative gonioscopy, and identified a negative correlation between blood reflux and IOP, with a lower IOP inducing better filling of SC. Furthermore, Gong and Francis (26) demonstrated that impaired and interrupted blood reflux into $\mathrm{SC}$ was consistent with a decrease in the number of quadrants with fluorescein egress into the episcleral veins via collector channels, and that blockage of the collector channel ostia occurred in patients with POAG. It is reasonable to assume that prompt reflux of blood into the canal and good episcleral filling reflects a patent canal and healthy collector channels, and suggests that the major problem of outflow resistance lies in the TM.

Although blood regurgitation occurs in the reverse direction of the natural aqueous humour passage, it may indicate that the canal is not collapsed, or at least that there are no adhesions present between the outer and inner wall. This also indicates that it is unlikely that resistance to aqueous outflow is present in the distal outflow facility beyond the SC. This allows the blood to fill the canal, which may obviously occur through the TM (the site that most likely contributes to aqueous outflow resistance). Consequently, a significant reduction in IOP is achieved after SLT, particularly in eyes with complete circumferential filling. The present study analysed the association between the number of quadrants in which blood reflux appeared and the decrease in IOP. The clear correlation between the quadrants with blood reflux in SC and the percentage decrease in IOP strongly supports this assumption. However, the present study had certain limitations. A total of 35 eyes from 25 POAG patients who presented at our department between March 2015 and November 2015 were included. This time window was short and the sample size was small. The drugs used by the patients prior to and after the treatment were recorded as part of the study, but it was not possible to perform a quantitative analysis of the association between the drug intake and the reduction of IOP after SLT. These factors require further study.

In conclusion, the present study indicated that blood reflux in SC had a favourable effect on the IOP after SLT and the number of blood reflux quadrants was inversely correlated with the post-SLT IOP. Blood reflux in SC may provide an indication of the functional status of the SC and the distal outflow facility. As the eyes with positive blood reflux had a more persistent significant IOP reduction and higher success rates after SLT, this diagnostic method may help to identify patients who may benefit the most from SLT. 


\section{Acknowledgements}

Not applicable.

\section{Funding}

This study was supported by the National Natural Science Foundation of China (grant no. 81770921).

\section{Availability of data and materials}

The analyzed data sets generated during the study are available from the corresponding author on reasonable request.

\section{Authors' contributions}

JG and SA performed the experiments. YZ and HZ conceived the study.

\section{Ethical approval and consent to participate}

The study was approved by the institutional ethics committees of Tongji Hospital, Huazhong University of Science and Technology (Wuhan, China; Trial approval number, K-2014-013). Informed consent was obtained from each patient prior to the start of the study.

\section{Consent for publication}

Not applicable.

\section{Competing interests}

The authors declare that they have no competing interests.

\section{References}

1. Quigley HA: Glaucoma. Lancet 377: 1367-1377, 2011.

2. Tamm ER and Fuchshofer R: What increases outflow resistance in primary open-angle glaucoma? Surv Ophthalmol 52 (Suppl 2): S101-S104, 2007.

3. Bill A and Svedbergh B: Scanning electron microscopic studies of the trabecular meshwork and the canal of Schlemm-an attempt to localize the main resistance to outflow of aqueous humor in man. Acta Ophthalmol (Copenh) 50: 295-320, 1972.

4. Latina MA and Park C: Selective targeting of trabecular meshwork cells: In vitro studies of pulsed and CW laser interactions. Exp Eye Res 60: 359-371, 1995.
5. Johnson DH and Johnson M: How does nonpenetrating glaucoma surgery work? Aqueous outflow resistance and glaucoma surgery. J Glaucoma 10: 55-67, 2001.

6. Leahy KE and White AJ: Selective laser trabeculoplasty: Current perspectives. Clin Ophthalmol 9: 833-841, 2015.

7. Waisbourd M and Katz LJ: Selective laser trabeculoplasty as a first-line therapy: A review. Can J Ophthalmol 49: 519-522, 2014.

8. De Keyser M, De Belder M, De Belder S and De Groot V: Where does selective laser trabeculoplasty stand now? A review. Eye Vis (Lond) 3: 10, 2016.

9. Wong MO, Lee JW, Choy BN, Chan JC and Lai JS: Systematic review and meta-analysis on the efficacy of selective laser trabeculoplasty in open-angle glaucoma. Surv Ophthalmol 60: 36-50, 2015.

10. McAlinden C: Selective laser trabeculoplasty (SLT) vs. other treatment modalities for glaucoma: Systematic review. Eye (Lond) 28: 249-258, 2014.

11. Kronfeld PC: Further gonioscopic studies on the canal of Schlemm. Arch Ophthal 41: 393-405, 1949.

12. Grieshaber MC: Channelography and mechanism of action in canaloplasty. Ophthalmologe 112: 319-324, 2015 (In German).

13. Grieshaber MC, Pienaar A, Olivier J and Stegmann R: Clinical evaluation of the aqueous outflow system in primary open-angle glaucoma for canaloplasty. Invest Ophthalmol Vis Sci 51: 1498-1504, 2010.

14. Francis BA, Loewen N, Hong B, Dustin L, Kaplowitz K, Kinast R, Bacharach J, Radhakrishnan S, Iwach A, Rudavska L, et al: Repeatability of selective laser trabeculoplasty for open-angle glaucoma. BMC Ophthalmol 16: 128, 2016.

15. Li M, Song Y, Zhao Y, Yan X and Zhang H: Influence of exercise on the structure of the anterior chamber of the eye. Acta Ophthalmol 96: e247-e253, 2018.

16. Coleman AL and Miglior S: Risk factors for glaucoma onset and progression. Surv Ophthalmol 53 (Suppl 1): S3-S10, 2008.

17. Bhartiya S, Ichhpujani $P$ and Shaarawy T: Surgery on the trabecular meshwork: Histopathological evidence. J Curr Glaucoma Pract 9: 51-61, 2015.

18. Ethier CR, Coloma FM, Sit AJ and Johnson M: Two pore types in the inner-wall endothelium of Schlemm's canal. Invest Ophthalmol Vis Sci 39: 2041-2048, 1998

19. Johnson MC and Kamm RD: The role of Schlemm's canal in aqueous outflow from the human eye. Invest Ophthalmol Vis Sci 24 : 320-305, 1983.

20. Weinreb RN, Leung CK, Crowston JG, Medeiros FA, Friedman DS, Wiggs JL and Martin KR: Primary open-angle glaucoma. Nat Rev Dis Primers 2: 16067, 2016.

21. Lee DA and Higginbotham EJ: Glaucoma and its treatment: A review. Am J Health Syst Pharm 62: 691-699, 2005.

22. Kennedy JB, SooHoo JR, Kahook MY and Seibold LK: Selective laser trabeculoplasty: An update. Asia Pac J Ophthalmol (Phila) 5: 63-69, 2016.

23. Phelps CD, Asseff CF, Weisman RL, Podos SM and Becker B: Blood reflux into Schlemm's canal. Arch Ophthalmol 88: 625-631, 1972.

24. Phelps CD: Arterial anastomosis with Schlemm's canal: A rare cause of secondary open-angle glaucoma. Trans Am Ophthalmol Soc 83: 304-315, 1985 .

25. Grieshaber MC: Ab externo Schlemm's canal surgery: Viscocanalostomy and canaloplasty. Dev Ophthalmol 50: 109-124, 2012.

26. Gong H and Francis A: Schlemm's Canal and Collector Channels as Therapeutic Targets. In: Surgical Innovations in Glaucoma. Samples JR and Ahmed IIK (eds). Springer, New York, NY, pp3-25, 2014. 